molecular and infrared emissions; these correlations are not too surprising because the infrared luminosity is thought to arise as black-body radiation from warm dust grains mixed within dense clouds of interstellar material.

Sanders et al. consider these observations to be overwhelming evidence that the phenomenon of ultraluminous infrared galaxies is a consequence of collisions between individual galaxies. Furthermore, given that these same galaxies have nuclear quasars, they argue that this process must be fundamentally related to the origin of quasars. They conclude that quasars are spawned by the collision of two gas-rich galaxies, and that the increased frequency of such collisions in a younger, denser Universe naturally explains why quasars were more conspicuous in the past.

These ideas are not completely new, as earlier observers had noted that a surprising number of quasars discovered optically have interacting galaxy companions. What is unique about the hypothesis put forward by the infrared astronomers is their contention that most quasars begin as ultraluminous infrared sources, and that optical observers cannot see them until the intense radiation has driven away the dust cocoon that shrouds the quasar birth. Sanders et al. make some preliminary suggestions as to where their individual galaxies lie in such an evolutionary sequence, and they are undertaking more observations to describe better the transition from infrared to optical quasar.

Now that it is clearly demonstrated that infrared-selected samples can reveal important aspects of quasars, fundamental questions must be addressed. What is the ultimate source of the energy heating the dust to make such ultraluminous infrared sources? How much of this energy arises within the non-thermal nucleus and how much within starbursts inside the molecular clouds around the nucleus? Is a quasar formed where none existed before as a consequence of the galaxy collison, or does a previously dormant black hole in a galaxy nucleus simply flare up as a new supply of gas is dumped into its vicinity? Were all early quasars so shrouded that optical surveys could never find the new quasars in the young (distant) Universe? And perhaps most puzzling, where does all of the dust come from that is necessary to explain the infrared radiation?

For the time being, further progress can only come using ground-based observations to target the galaxies found and sorted by IRAS. No further help from new space observations will come until the launch of the Infrared Space Observatory (ISO), expected in 1992.

Daniel W. Weedman is in the Department of Astronomy, Pennsylvania State University, University Park, Pennsylvania 16802, USA.

\title{
Seismology
}

\section{The deep roots of continents}

\section{Thorne Lay}

Continents are the Earth's most conspicuous structures; their precise role in the dynamic evolution of the planet, however, remains elusive. Early notions of continental drift, motivated by observations requiring large-scale translation of continents, were repeatedly stymied by the lack of a mechanism capable of propelling relatively thin $(30 \mathrm{~km})$ continental masses through or over the globally extensive oceanic crust. The subsequent theories of seafloor spreading and plate tectonics, which treat the oceanic plates as the upper thermal boundary layer of a mantle convection system, allow the embedded continental nuggets to drift as the boundary layer circulates. It is conventionally asserted that any thermal or compositional differences between the mantle underlying continents and that underlying oceans vanish below depths of 150-200 $\mathrm{km}$. Lerner-Lam and Jordan', however, now provide strong evidence that relatively fast seismic velocities under stable continental regions extend more than 220 $\mathrm{km}$ into the mantle.

There is no question that continents are chemically differentiated, buoyant structures that resist recycling down into the mantle, and hence strongly influence the kinematics of surface plates; but the controversial assertion that the stable continental shields have roots extending as much as $400 \mathrm{~km}$ into the mantle has radical implications for the dynamic role of continents in plate tectonics.

Jordan $^{2}$ first advanced the thick-plate model for continents, basing it primarily on observations of seismic body and surface waves that he interpreted as evidence for fast seismic velocities underlying continents to depths of $400 \mathrm{~km}$ or more. For many years controversy surrounded this interpretation, and new analyses have only recently enhanced the resolution of the vertical extent of seismic heterogeneity.

The best resolution of the upper-mantle seismic-velocity structure is obtained by analysing groups of seismic waves that bottom at different depths in the mantle, including waves that are multiply reflected off the surface. The waves can either be analysed as discrete body-wave arrivals ${ }^{3,4}$ or as interfering surface-wave overtones ${ }^{1,5,6}$.

Lerner-Lam and Jordan in their new work ${ }^{i}$ adopt the latter approach for fundamental and higher-mode 'Rayleigh' wave arrivals traversing either old oceanic or stable continental paths. The variety of Earth models obtainable from sparse seismic-wave data precludes an absolute determination of separate upper-mantle velocity structures under continents and oceans. Thus the authors combine a hypothesis-testing procedure with the structure inversions to focus explicitly on the required depth extent of lateral variations between the two types of region. They find that lateral variations in the velocity structure of primary $(\mathrm{P})$ waves can be confined to the uppermost $220 \mathrm{~km}$ of the mantle; lateral variations in the secondaryor shear-wave (S) velocity structure, however, must extend deeper, with satisfactory models having significant differences between oceanic and continental regions as deep as $400 \mathrm{~km}$. Similar variations in S-velocity structure have been obtained in previous studies ${ }^{2-5}$, but the lack of any formal analysis of the resolution has always left the models' reliability uncertain.

Most seismic evidence seems to support the contention that differences of shear velocity between mantle beneath continents and that beneath oceans, extend more than $220 \mathrm{~km}$ into the upper mantle. But even having accepted this, the interpretation of this heterogeneity and its dynamic implications is still controversial. Anderson? emphasizes the intrinsic ambiguity of interpreting relative velocity variations, arguing that the mantle more than $200 \mathrm{~km}$ under continental shields is normal unmolten mantle, whereas the hotter suboceanic mantle has anomalously low shear velocities because of partial

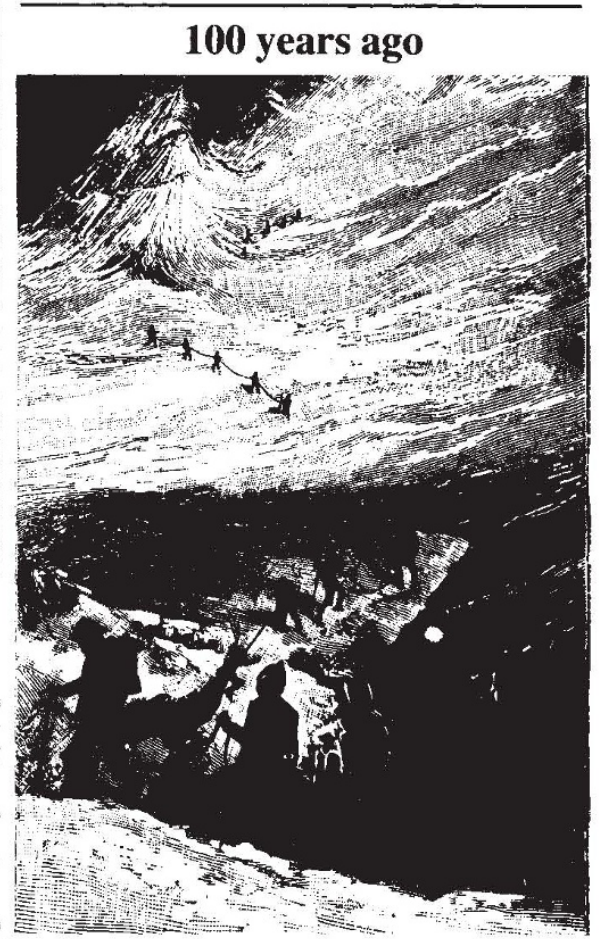

M. Richard's team proved that it was possible to live at high altitude on Mont Blanc.

From Nature 38, 35; 10 May 1888. 